\title{
THE ARTHROSCOPIC TREATMENT OF CHONDROMALACIA PATELLAE
}

\author{
D. J. OGILVIE-HARRIS, R. W. JACKSON
}

From Toronto Western Hospital and the University of Toronto

\begin{abstract}
Three hundred and nineteen patients who had chondromalacia patellae and persistent patellofemoral pain after six months of conservative management underwent arthroscopy and arthroscopic surgery. The results in four aetiological groups were reviewed at one year and five years after operation. Morbidity was minimal. Lavage produced early remission in all groups. Shaving offered a particular advantage in the posttraumatic group. Lateral release plus shaving and lavage was beneficial in the group with maltracking patellae and in half of the idiopathic group. In the group with unstable patellae, lateral release produced good results in only one in four patients. In conclusion, we consider that arthroscopic surgery has a useful role to play in the management of chondromalacia patellae.
\end{abstract}

The term chondromalacia patellae, although used as a synonym for patellofemoral pain, should ideally be reserved to describe pathological changes in the articular cartilage of the patella (Emery and Meachim 1973; Ficat and Hungerford 1977; Radin 1979; Insall 1982). Although the changes of chondromalacia patellae may be asymptomatic, in some instances patellofemoral pain persists despite a full range of conservative therapy (Karlson 1939; Bennett, Waine and Bauer 1942; Jackson 1976; Casscells 1978, 1979; Donohue et al. 1983). If such patients have symptoms severe enough to limit their activities significantly, then further diagnostic and surgical procedures are indicated.

In this paper we report the long-term results of treatment of those patients whose persistent patellofemoral pain was clearly due to chondromalacia patellae. We excluded from the study those whose symptoms were attributable to other intra-articular pathology and those in whom chondromalacia patellae was an incidental finding.

In patients with persistent patellofemoral pain, we performed diagnostic arthroscopy followed by arthroscopic surgical procedures when indicated. We reviewed these patients at one year and at five years after operation. We tried to find out whether arthroscopic procedures were effective in relieving patellofemoral pain caused by chondromalacia patellae, and to determine the role of arthroscopic surgery in its management.

D. J. Ogilvie-Harris, FRCS(C), Orthopaedic Surgeon

R. W. Jackson, MD, MS, FRCS(C), Professor, Department of Surgery, University of Toronto, and Chief, Division of Orthopaedic Surgery

Toronto Western Hospital, Suite 311, 25 Leonard Avenue, Toronto, Ontario, Canada M5A 2R2.

Requests for reprints should be sent to Dr D. J. Ogilvie-Harris.

(C) 1984 British Editorial Society of Bone and Joint Surgery $0301-620 \mathrm{X} / 84 / 5132 \$ 2.00$

\section{METHODS}

Criteria for selection. In the management of the patient with patellofemoral pain, our first concern has been accurate diagnosis. Clinical diagnosis was based on the assessment of morphology, stability and tracking of the patella and on the general function of the patellofemoral joint (Insall 1982). Radiological diagnosis was based on $30^{\circ}$ tangential views of the patella in addition to standard radiographs (Laurin, Dussault and Levesque 1979). When we considered that the symptoms and signs were those of patellofemoral dysfunction, our initial treatment was conservative - a course of physiotherapy and therapeutic doses of aspirin. The physiotherapy consisted of static quadriceps exercises at full extension and isotonic quadriceps exercises from $30^{\circ}$ of flexion to full extension.

In those patients with persistent symptoms despite six months of conservative treatment, we performed arthroscopy and the definitive diagnosis of chondromalacia patellae was based on the arthroscopic findings. We used the term chondromalacia patellae when there was chondrosis, that is softening or derangement of the articular cartilage without significant subchondral bony changes (Ficat and Hungerford 1977). Thus we distinguished patellofemoral chondrosis from patellofemoral arthrosis, in which there are significant signs of bony reaction in the form of subchondral sclerosis, osteophytes, cyst formation and other changes of osteoarthritis (Radin 1979; Insall 1982).

Arthroscopic assessment. Arthroscopy was performed through a standard anterior inferolateral portal, and a probe was used to assess the articular surface in all cases (Insall, Falvo and Wise 1976). We graded the chondromalacia patellae (Jackson 1976) as follows (Fig. 1). Grade I: Softening of the articular cartilage upon probing, minor surface fissuring and the classical blister lesionthe "closed chondrosis" of Ficat (Goodfellow, Hungerford and Woods 1976; Ficat and Hungerford 1977). 


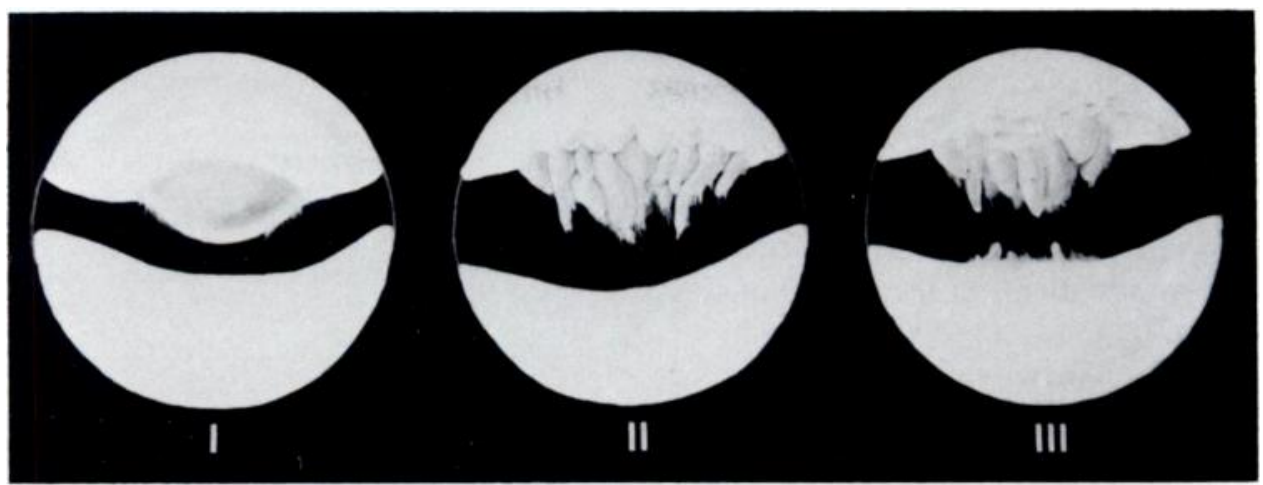

Fig. 1

Arthroscopic grading of chondromalacia patellae. Grade I, blister lesion; Grade II, fasciculation; Grade III, exposure of subchondral bone with changes on the femoral groove.

Grade II: Major fasciculation of the articular cartilage confined to the patella - the "crab meat" appearance or "open chondrosis" of Ficat and Hungerford. Group III: Exposure of the subchondral bone of the patella with surface changes in the femoral groove.

Operative procedures. Three separate arthroscopic procedures were performed-lavage, shaving and lateral release. Formal lavage was carried out by irrigating the joint with two to three litres of saline through the arthroscope sheath. Shaving of the damaged area of cartilage was performed with a powered intra-articular shaver or with hand instruments such as a pituitary rongeur. Care was taken to excise only the damaged cartilage and to leave as smooth a surface as possible; this excision was not usually carried down to the subchondral bone, and the subchondral bone plate was not penetrated. After shaving the damaged area, the cartilaginous fragments were removed from the joint by suction or by repeated lavage.

Lateral release was performed through a small lateral incision using special scissors under arthroscopic control. The lateral retinaculum and capsule were cut, taking care to release the attachments at the superolateral corner of the patella. After the release, a compressive bandage and a sponge were placed over the lateral border of the patella and retained for 10 days.

All these procedures were performed on an outpatient basis under general anaesthesia. Occasionally, a patient was admitted to hospital when general medical conditions indicated an increased anaesthetic risk.

Postoperative assessment. We decided to classify our results simply as good or bad, based entirely on the patient's subjective rating. A result was considered good if the patient had little or no discomfort and could pursue all desired activities. A result was considered poor if the patient had significant discomfort such as to limit the ability to perform desired activities.

We reviewed the records of patients who had had arthroscopic surgery for chondromalacia patellae in the 10 -year period 1970 to 1980 ; in one-third the records were adequate for assessment; in the remaining twothirds we contacted the patients for follow-up.

\section{Aetiology}

We divided the patients into four aetiological groups.

The maltracking patellae. These patients had the "excess lateral pressure syndrome" of Ficat (Goodfellow et al. 1976). They demonstrated clinically abnormal tracking of the patella. The lateral facet was tender to compression. They often had palpable thickening of the lateral retinaculum and tenderness at the superolateral border of the patella. The Q-angle (Insall et al. 1976) measured more than $20^{\circ}$ with the knee flexed to $30^{\circ}$. Radiographs demonstrated lateral tilt of the patella and other signs as described by Ficat and Hungerford (1977). There were 63 patients in this group at the one-year review $(19.8 \%$ of the total).

The unstable patellae. Patients with one or more episodes of dislocation and with patellofemoral pain were included in this group, which also included those with demonstrable subluxation either clinically or under general anaesthesia at the time of arthroscopy. We excluded patients who had had dislocations but who did not have persistent patellofemoral pain, and those in whom chondromalacia patellae was not found at arthroscopy. There were a few cases of patella alta in this group, but these patients also had demonstrable subluxation. Fiftytwo patients $(16.3 \%$ of the total) fell into this group at one year.

Post-traumatic chondromalacia. Inclusion in this group depended on a clear-cut history of direct injury to the patella, with symptoms definitely following the injury. Patients who had had symptoms before the injury were excluded even though we recognise that trauma may exacerbate pre-existing disease. This group comprised 45 patients $(14.1 \%$ of the total). These patients had either fallen directly onto their knees or had been involved in motor vehicle accidents and impacted their knees on the interior of the vehicle. They had normal alignment and stability of the patellofemoral joint.

Idiopathic chondromalacia patellae. This was by far the largest group consisting of 159 patients $(49.8 \%$ of the total). We could find no cause for the disease in these patients, and were unable to attribute their disease to any of the three categories described. 


\section{RESULTS}

Over the chosen 10-year period, 354 patients underwent arthroscopic surgery for chondromalacia patellae producing patellofemoral pain; $319(90 \%)$ were available for follow-up one year after operation and $272(85 \%$ of the latter) at five years. The ages of the patients varied from 17 to 42 years with most patients in the second or third decades. There were $57 \%$ females and $43 \%$ males.

The maltracking patellae. There were 63 patients in our one year follow-up and 56 at five years. At arthroscopy, we found the chondromalacia was confined to the lateral facet of the patella. Grade I chondromalacia was found in 17 patients $(27 \%)$, Grade II in 40 patients $(63 \%)$ and Grade III in $6(10 \%)$. All patients with maltracking patellae were treated by arthroscopic shaving, lavage and lateral release. At arthroscopy, we could demonstrate the abnormal tilt of the patella and the abnormal lateral tracking. Lateral release appeared to correct the abnormal tilt and to improve tracking (Figs 2 and 3).

Of those who had Grade I chondromalacia, $88 \%$ had a good result at one year; this figure was almost unchanged at five years with $85 \%$ good results (Table I). Although Grade II had $87 \%$ good results at one year, the number of good results dropped to $65 \%$ by five years. Grade III initially showed $67 \%$ good results but by five years only $20 \%$ were still good. Thus, patients with Grade I changes had a good chance of obtaining and maintaining a successful result; Grade II had a good chance of obtaining

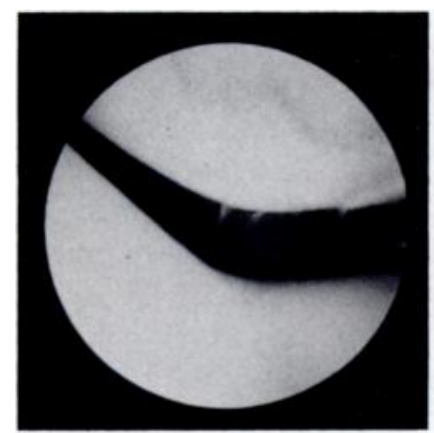

Fig. 2

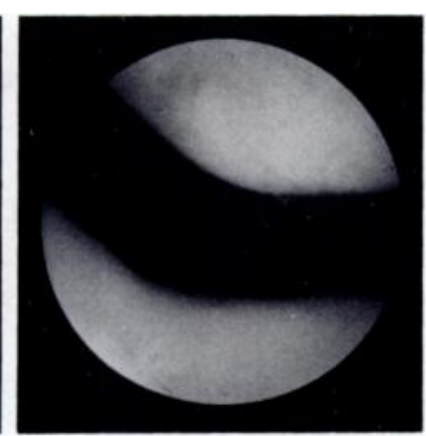

Fig. 3
Figure 2-Arthroscopic demonstration of abnormal patellar tilt seen in a case of maltracking patella. Figure 3-Correction after a lateral release.

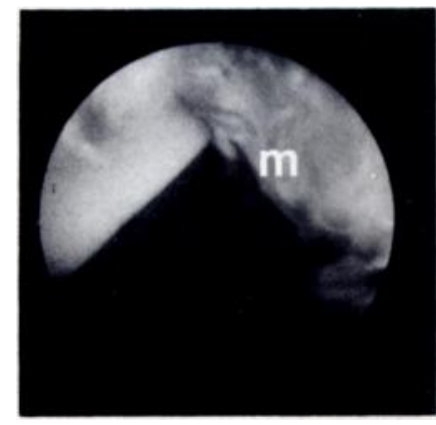

Fig. 4

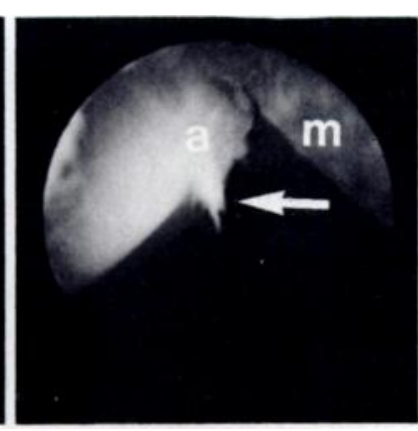

Fig. 5
Arthroscopic demonstration of the method of damage to the medial facet of the patella $(a)$ as it crosses the lateral femoral condyle $(m)$ Arrow shows direction of dislocation. Figure 4-Before dislocation. Figure 5-After dislocation.
Table I. Good results in the group with maltracking patellae

\begin{tabular}{|cccccc|}
\hline & At one year & & & At five years \\
\cline { 2 - 3 } \cline { 6 - 6 } Grade & Number & $\%$ & & Number & $\%$ \\
\hline I & 15 of 17 & 88 & & 12 of 14 & 85 \\
II & 35 of 40 & 87 & & 24 of 37 & 65 \\
\cline { 6 - 7 } & 4 of 6 & 67 & & 1 of 5 & 20 \\
\hline
\end{tabular}

Table II. Good results in the group with unstable patellae

\begin{tabular}{|c|c|c|c|c|c|}
\hline \multirow[b]{2}{*}{ Grade } & \multirow[b]{2}{*}{ Procedure } & \multicolumn{2}{|c|}{ At one year } & \multicolumn{2}{|c|}{ At five years } \\
\hline & & Number & $\%$ & Number & $\%$ \\
\hline I & $\mathbf{R}+\mathbf{L}$ & 3 of 3 & 100 & 3 of 3 & 100 \\
\hline II & $\begin{array}{l}S+L \\
R+S+L\end{array}$ & $\begin{array}{l}7 \text { of } 20 \\
6 \text { of } 11\end{array}$ & $\begin{array}{l}35 \\
55\end{array}$ & $\begin{array}{l}5 \text { of } 18 \\
3 \text { of } 9\end{array}$ & $\begin{array}{l}28 \\
33\end{array}$ \\
\hline III & $\begin{array}{l}S+L \\
R+S+L\end{array}$ & $\begin{array}{l}4 \text { of } 13 \\
2 \text { of } 5\end{array}$ & $\begin{array}{l}30 \\
40\end{array}$ & $\begin{array}{l}3 \text { of } 12 \\
1 \text { of } 4\end{array}$ & $\begin{array}{l}25 \\
25\end{array}$ \\
\hline
\end{tabular}

R, release S, shaving L, lavage

a good initial result but less chance of sustaining it; and Grade III had a reasonable chance of early relief of symptoms, but only a small chance of sustaining it.

The unstable patellae. There were 52 patients in our oneyear follow-up and 46 at five years. At arthroscopy, we found that the chondromalacia was usually on the medial facet. We could show at arthroscopy that the damage to the medial facet occurred as the patella subluxated or dislocated over the lateral femoral condyle (Figs 4 and 5). Grade I chondromalacia was found in 3 patients $(6 \%)$, Grade II in 31 patients (60\%) and Grade III in $18(34 \%)$. Thirty-three patients $(63 \%)$ had arthroscopic shaving and lavage and $16(31 \%)$ had arthroscopic shaving, lavage and lateral release. The three patients with Grade I chondromalacia had a lateral release and lavage only.

All the patients with Grade I chondromalacia had a good result at both one year and five years (Table II). The one-year results in Grade II show that those patients who had had shaving, lavage and lateral release had a higher percentage of good results $(55 \%)$ than those who had had only shaving and lavage (35\%); however, there was only a slight difference between the results at five years (Fig. 6). Hence although lateral release seemed to offer an advantage in producing a higher percentage of good results at one year, this initial improvement was not sustained for the five-year period. With Grade III chondromalacia there was little difference between those who had had shaving and lavage, and those who had also had a lateral release; by five years only $25 \%$ of patients with Grade III chondromalacia still had a good result (Fig. 6).

Post-traumatic chondromalacia. At one year, 45 patients met the criteria for inclusion in this group. Three patients had lavage only and these are not considered further. Of the remaining 42, Grade I chondromalacia was found in 


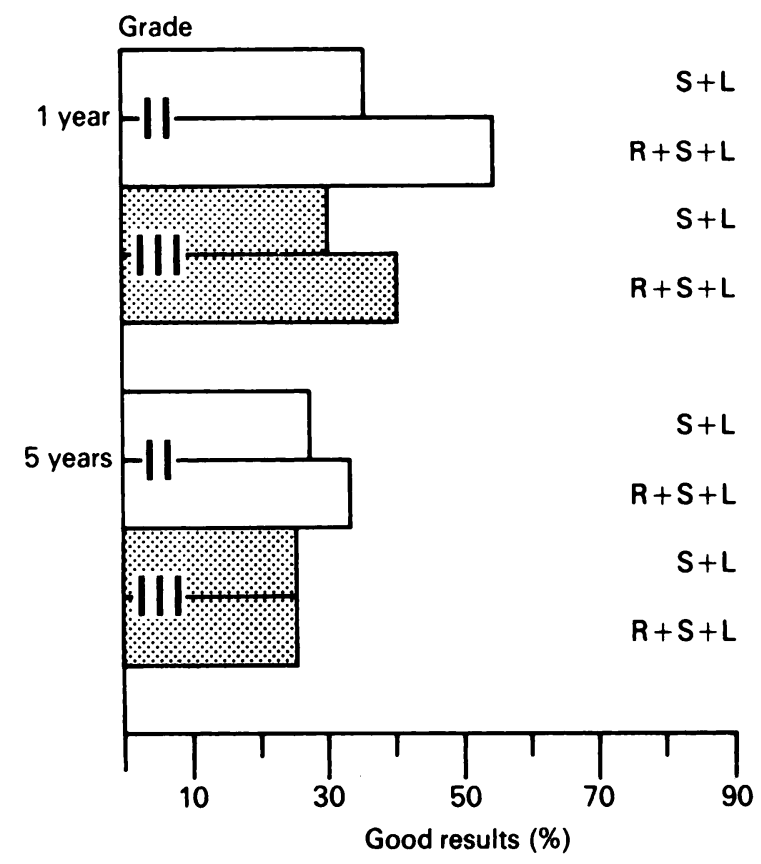

Fig. 6

Results in the unstable patella group-comparison at one and five years. Treatment: $R$, lateral release; $S$, shaving; $L$, lavage.

7 patients $(17 \%)$, Grade II in 28 patients $(67 \%)$ and Grade III in $7(17 \%)$. The lesion was located on the medial facet and central area in 29 patients $(69 \%)$, at the inferior pole in 2 patients $(5 \%)$ and on the lateral facet in 11 patients ( $26 \%$ ). All 42 patients had arthroscopic shaving to remove the damaged area, followed by lavage; five were lost to follow-up, so 37 were available for the five-year assessment.

Those with Grade I had a high percentage of good results at one year $(86 \%)$ (Table III); this was sustained at the five-year assessment ( $83 \%$ ). Grade II had a slightly lower percentage of good results both at one year $(79 \%)$ and at five years $(73 \%)$. Although three out of seven patients $(43 \%)$ with Grade III chondromalacia had a good result at one year, only one out of five $(20 \%)$ had a good result at five years. These results indicate that patients with Grade I and II chondromalacia of traumatic aetiology can expect a high percentage of good long-term results, but those with Grade III have only a small chance of arthroscopic surgery being successful.

Idiopathic chondromalacia. The 159 patients in the oneyear assessment were reduced to 133 in the five-year assessment, an $84 \%$ follow-up rate. Grade I chondromalacia was found in 16 patients $(10 \%)$, Grade II in 118 patients $(74 \%)$ and Grade III in $25(16 \%)$. One hundred and twelve $(70 \%)$ of the lesions were located on the medial facet, $16(10 \%)$ on the inferior pole, and $31(20 \%)$ on the lateral facet. Twenty-four patients $(15 \%)$ had lavage alone, $97(61 \%)$ had a shaving procedure and 38 $(24 \%)$ had shaving and lateral release.

Those patients with Grade I had lavage alone; $88 \%$ had a good result at one year and $82 \%$ at five years (Table IV). Whether the same results at five years would have

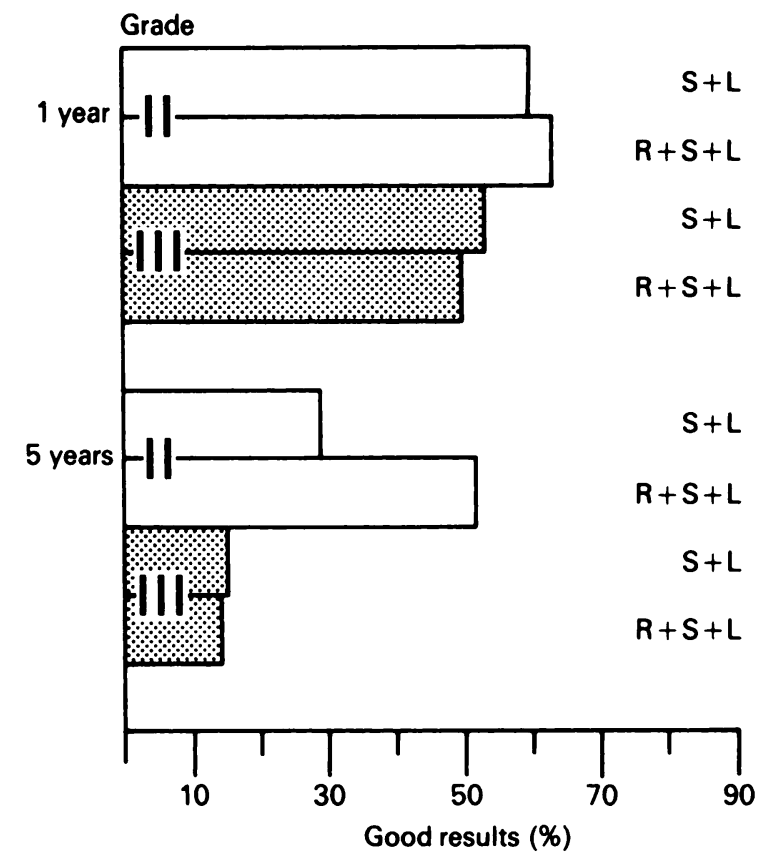

Fig. 7

Idiopathic group-comparison of results at one and five years. $R$, release; $S$, shaving; $L$, lavage.

been obtained without any intervention remains a matter of conjecture.

The results of Grade II chondromalacia after shaving and lavage were good at one year, and much the same with lateral release $(63 \%)$ or without $(60 \%)$. However, at five years, those who had had a lateral release had better results $(52 \%)$ than those who had had shaving and lavage alone $(29 \%)$. Interestingly enough, those patients in Grade II who had lavage alone had as good a result at one year $(63 \%)$ as those who had the other two procedures; and lavage alone was almost as good $(28 \%)$ as shaving after five years $(29 \%)$. However, the results of lavage

Table III. Good results in the group with post-traumatic patellae

\begin{tabular}{|cccccc|}
\hline & At one year & & At five years \\
\cline { 2 - 3 } \cline { 5 - 6 } Grade & Number & $\%$ & & Number & $\%$ \\
\hline I & 6 of 7 & 86 & 5 of 6 & 83 \\
II & 22 of 28 & 79 & 19 of 26 & 73 \\
III & 3 of 7 & 43 & & 1 of 5 & 20 \\
\hline
\end{tabular}

Table IV. Good results in the idiopathic group

\begin{tabular}{|clccccc|}
\hline & & \multicolumn{2}{c}{ At one year } & & \multicolumn{2}{c|}{ At five years } \\
\cline { 7 - 7 } \cline { 7 - 7 } Grade & Procedure & Number & $\%$ & & Number & $\%$ \\
\hline I & L & 14 of 16 & 88 & 9 of 11 & 82 \\
II & L & 5 of 8 & 63 & & 2 of 7 & 28 \\
& S + L & 48 of 80 & 60 & 21 of 72 & 29 \\
& R + S + L & 19 of 30 & 63 & 12 of 23 & 52 \\
III & S + L & 9 of 17 & 53 & & 2 of 13 & 15 \\
& R + S + L & 4 of 8 & 50 & & 1 of 7 & 14 \\
\hline
\end{tabular}


alone at five years $(28 \%)$ were inferior to those of lateral release $(52 \%)$. It would seem, therefore, that the combination of lateral release, shaving and lavage offered a distinct advantage at five years to patients with Grade II chondromalacia.

In those with Grade III chondromalacia, lateral release seemed to offer no advantage over shaving either at one year or at five years. The results looked encouraging at one year $(50 \%)$, but by five years were dismal $(14 \%)$.

The only patients with idiopathic chondromalacia to benefit significantly from our surgical procedures appeared to be those with Grade II chondromalacia in whom lateral release plus shaving and lavage produced a high percentage of good long-term results (Fig. 7).

\section{Complications}

There were no major complications such as infection or deep venous thrombosis. However, four of the patients who had lateral release developed large effusionspresumably haemarthroses; these settled without further intervention.

\section{DISCUSSION}

Results in relation to severity of disease. The overall results clearly show that the grading system we have used is closely related to the long-term prognosis. In each group, Grade I had the best results, Grade II intermediate results and Grade III the worst results. Such a conclusion may well have been predicted as the grades reflect a progressively greater degree of damage to the patellofemoral joint.

Our grading system is easy to use in clinical practice with clear cut-off points between each grade. It correlates well with the patho-anatomical staging system of Outerbridge (1961). However, we distinguished chondrosis from arthrosis as have others (Ficat and Hungerford 1977; Radin 1979; Insall 1982).

In general, the results of arthroscopic surgery in Grade I chondromalacia were excellent. In all probability the major contribution to the high remission rate was the lavage. The evidence for this is the fact that even with advanced chondromalacia (Grade III), a high percentage of patients had good early results; these were, however, not sustained over the five-year period. In these advanced cases, lavage produced good early results, but as the underlying pathology was not corrected, the long-term results were poor. Furthermore, in Grade I idiopathic chondromalacia good results were obtained from lavage alone, without the addition of further procedures. Hence, assessment of this particular subgroup demonstrated the effect of lavage alone in producing a high remission rate.

In Grade II chondromalacia, the early results at one year were almost as good as the Grade I results; this remission of symptoms may be ascribed to the lavage. At five years, however, those patients who had had lavage alone did not fare so well as those in whom it had been combined with other arthroscopic procedures. Hence, in order to maintain the initial good results, some alteration in the pathomechanics of the patellofemoral joint was necessary.

In Grade III chondromalacia, the five-year results were poor-an average of less than $20 \%$ had a good result. However, about half the patients had relief of symptoms at one year; this temporary relief again was probably the effect of the lavage.

Results in relation to aetiology. It seems clear that trauma, instability, or maltracking (Larson et al. 1978) can cause chondromalacia patellae.

In our series, Grades I and II chondromalacia with a maltracking patella could be adequately treated by arthroscopic shaving, lavage and lateral release. The high percentages of good results relate to the ability to correct the abnormal tracking forces through release of the contracted lateral bands and retinaculum. The poor results in Grade III reflect the advanced state of the disease.

The unstable patella with chondromalacia could be adequately treated only in Grade I. In Grade II only onethird of patients had long-term relief of symptoms. We feel the underlying reason is that lateral release and shaving cannot correct the basic instability. However, as one-third of patients do sustain a good long-term result, we recommend arthroscopy and lateral release as an initial procedure; the other two-thirds may well require further surgery, and should be so informed. Nonetheless, one-third will have good long-term results and this is worthwhile when obtained with minimal risk and morbidity. In Grade III the results were poor because arthroscopic surgery cannot correct the advanced pathology. One in four patients, however, do achieve longterm relief and are therefore spared major reconstructive surgery; thus, even though the success rate is low, the procedures described may be justified as a first step.

Post-traumatic chondromalacia responded well to shaving, which removed the damaged area of cartilage. As the pathological changes follow a single incident, once the offending area is removed presumably the disease should not progress. This presumption is borne out by the results with Grades I and II chondromalacia. However, with Grade III the results at five years were not good because the disease was too advanced. It would seem that leaving the damaged area for a long period of time may lead to progression to Grade III, thereby precluding a good result.

Idiopathic chondromalacia was the largest group, constituting half of our patients; this reflects the lack of adequate knowledge regarding the aetiology of the disease. In Grade I, good remission rates were obtained with lavage alone. These patients did not seem to deteriorate significantly over the five-year period and we do not feel that the results at five years can be ascribed to the effects of lavage alone. Treatment is almost certainly responsible for the early remission, as these patients had had significant symptoms for at least six months before 
operation, and had relief soon after. Long-term remission may be due to the fact that the disease does not progress rapidly beyond Grade I.

In Grade II the results of lavage were equal to those of shaving combined with lavage; hence the shaving itself was of no detectable benefit. However, the addition of lateral release nearly doubled the percentage of good results at five years; hence in Grade II chondromalacia, alteration of the biomechanics of the joint seems to improve long-term results significantly. In Grade III chondromalacia the results were much the same as in the other aetiological groups - that is, there was a good initial result but a poor long-term result.

The rationale of treatment. The reasons for the success of arthroscopic surgery are still a matter for conjecture. There is good evidence in the literature that exposure of the deeper layers of articular cartilage and the presence of cartilaginous debris produces synovitis. Such synovitis can be seen in patients with open chondrosis (Grades II and III chondromalacia patellae). It can be reproduced experimentally by the introduction of cartilaginous debris and proteoglycans into joints (Chrisman, Fessel and Southwick 1965; Dingle et al. 1979; Donohue et al. 1983). We postulate that in chondromalacia patellae intermittent shedding of debris produces episodes of synovitis. Intermittent effusions and "flares" are common in patients with chondromalacia patellae. Lavage removes this debris and hence produces an early remission; but it does nothing more, so that the relief of symptoms is often only temporary.

Shaving attempts to remove or to smooth the area of abnormal cartilage (Wiles, Andrews and Bremner 1960). In the post-traumatic cases, it seemed to produce good results which were sustained. Presumably removal of the damaged area stopped further shedding of debris and in addition removed an abnormal area of stress on the subchondral bone (Donohue et al. 1983). In the other three groups-maltracking, unstable and idiopathicshaving seemed to offer little advantage. It may remove already damaged cartilage and may hasten remission, but it did not prevent progression of the underlying disease.

Lateral release alters the pathomechanics of the patellofemoral joint. When abnormal tracking is the major cause of recurrent cartilaginous trauma, then lateral release can correct the abnormal mechanicshence our good results in patients with the excess lateral pressure syndrome or with maltracking patellae, which are mirrored by similar results in the literature. Where results very different from ours are reported, it seems likely that the patients selected for lateral release were not specifically those with maltracking patellae (Insall 1982). However, our results with unstable patellae were poor-simple lateral release was not sufficient to correct the underlying pathology; hence, repeated damage to the patellae occurred with progression of the disease. It is pertinent to point out that these observations apply only to unstable patellae with chondromalacia - the results of lateral release in dislocating or subluxating patellae without chondromalacia were not included.

We would like to stress that most patients with patellofemoral pain can be treated conservatively and that the patients who underwent surgery were a small, highly selected group who had significant and persistent symptoms. In these patients, arthroscopic surgery offers substantial benefits with minimal risk and morbidity.

\section{REFERENCES}

Bennett GA, Waine H, Baver W. Changes in the knee joint at various ages: with particular reference to the nature and development of degenerative joint disease. New York: Commonwealth Fund, 1942.

Casscells SW. Gross pathological changes in the knee joint of the aged individual: a study of 300 cases. Clin Orthop 1978; 132:225-32.

Casscells SW. The arthroscope in the diagnosis of disorders of the patellofemoral joint. Clin Orthop 1979;144:45-50.

Chrisman OD, Fessel JM, Southwick WO. Experimental production of synovitis and marginal articular exostoses in knee joints of dogs. Yale $J$ Biol Med 1965;37:409-12.

Dingle JT, Saklatuala J, Hambry R, Tyler J, Fell HB, Jubb R. A cartilage catabolic factor from synovium. Biochem J 1979; 184:177-80.

Donohue JM, Buss D, Oegema TR Jr, Thompson RC Jr. The effects of indirect blunt trauma on adult canine articular cartilage. J Bone Joint Surg [Am] 1983;65-A:948-57.

Emery IH, Meachim G. Surface morphology and topography of patello-femoral cartilage fibrillation in Liverpool necropsies. J Anat 1973 ; 116: 103-20.

Goodfellow J, Hungerford DS, Woods C. Patello-femoral joint mechanics and pathology. 2: Chondromalacia patellae. J Bone Joint Surg [Br] $1976 ; 58-B: 291-9$.

Ficat RP, Hungerford DS. Disorders of patello-femoral joint. Paris: Masson, 1977.

Hughston JC. Subluxation of the patella. J Bone Joint Surg [ Am] 1968;50-A : 1003-26.

Insall J. Current concepts review : patella pain. J Bone Joint Surg [Am] 1982;64-A : 147-52.

Insall J, Falvo KA, Wise DW. Chondromalacia patellae: a prospective study. J Bone Joint Surg [ Am] 1976;58-A : 1-8.

Jackson RW. Surgery of the patellofemoral joint. Part III: Etiology of chondromalacia patellae. Am Acad Orthop Surg Instr Course Lect $1976 ; 25: 36-40$.

Karlson S. Chondromalacia patellae. Acta Chir Scand 1939;83:347-81.

Larson RL, Cabaud HE, Slocum DB, James SL, Keenan T, Hutchinson T. The patellar compression syndrome: surgical treatment by lateral retinacular release. Clin Orthop 1978;134:158-67.

Laurin CA, Dussault R, Levesque HP. The tangential X-ray investigation of the patellofemoral joint: X-ray technique, diagnostic criteria and their interpretation. Clin Orthop 1979; 144:16-26.

Outerbridge RE. The etiology of chondromalacia patellae. J Bone Joint Surg [Br] 1961;43-B:752-7.

Radin EL. A rational approach to treatment of patellofemoral pain. Clin Orthop 1979; 144:107-9.

Wiles P, Andrews PS, Bremner RA. Chondromalacia of the patella : a study of the later results of excision of articular cartilage. J Bone Joint Surg $[B r] 1960 ; 42-B: 65-70$. 